\title{
Zonotopic Set-Membership Estimation for Interval Dynamic Systems
}

\author{
V.T.H. Le, T. Alamo, E.F. Camacho, C. Stoica and D. Dumur
}

\begin{abstract}
This paper presents an improved method for guaranteed state estimation of discrete-time linear-time varying systems affected by disturbances, noises and structured uncertainties modeled as interval uncertainties. Under the hypothesis that the disturbances and the noises are bounded, a zonotopic outer approximation of the state estimation domain is computed offering good performance and low complexity compared to the existing methods. The size of this zonotope is decreased by solving an off-line optimization problem. The advantages of the proposed approach are illustrated via a numerical example.
\end{abstract}

\section{INTRODUCTION}

In the context of control systems theory, the system state is necessary to solve many control problems such as implementation of a feedback control or system diagnosis. Consequently, estimating the state of a dynamic system using available measurements is an important issue. The state estimation can be formulated as follows. Under the hypothesis of a mathematical model of a real dynamical process with some noisy measurements, the state of the real process has to be estimated. This can be generally difficult and time consuming as mathematical models are never perfect representations of real plants and the available measurements do not provide perfect data due to existing noises or sensors' limitations. In the literature, the approach of Kalman filter [1] appeared very early (from the 1960s) and it is still a widely applied technique. It is based on some probabilistic assumptions about the perturbations and the noises. The state estimation is done by minimizing the error variance. However these assumptions are sometimes not realistic and difficult to validate.

Another approach is the set-membership [2] or worst-case estimation. In the last 50 years, it has received increased attention from control researchers [3], [4], [5], [6] [7], [8], [9], [10]. Set-membership estimation relies on the assumption of unknown but bounded perturbations and noises without considering any other hypotheses on the distribution of perturbations and measurement noises. The state estimation set is characterized as a compact set containing all possible system states that are consistent with the uncertain system and the measurement noise. Similar to Kalman filter, this approach consists in 3 steps: the prediction step, the measurement step and the update or correction step. Using set-membership estimation approach results in a trade-off

V.T.H. Le, C. Stoica and D. Dumur are with SUPELEC Systems Sciences (E3S) - Automatic Control Department, 3 rue Joliot Curie, F-91192, Gif-sur-Yvette cedex, France (e-mail: \{vutuanhieu.le; cristina.stoica; didier.dumur\}@supelec.fr).

T. Alamo and E.F. Camacho are with Department of Ingeniería de Sistemas y Automática, Universidad de Sevilla, Camino de los Descubrimientos, 41092 Sevilla, Spain (e-mail: alamo@cartuja.us.es, eduardodesi.us.es). between the computation load and the size of the set. In order to exactly represent the domain of variation for the system state in a linear formulation, polytopes can be successfully used for a reasonable number of vertices of the polytopes [5]. In order to reduce the complexity due to a large number of vertices, ellipsoids [11], [8] has been firstly used to represent the estimation set, sometimes with a significant loss of performance. To improve the performance compared to ellipsoids and to reduce the computation time compared to polytopes, the representation by zonotopes is further used in this paper. Contrary to ellipsoids, the Minkowski sum of two zonotopes is a zonotope, this property being very useful in the prediction step of the state estimation. In addition, zonotopes can represent uncertainties independently in each direction of the state-space being a suitable solution for the elimination of the wrapping effect [12]. The state-space domain is represented by zonotopes in many applications such as: reachability analysis [13], [10], collision detection [14], states estimation [7], [9] and fault diagnosis [15], [16].

Most of the works cited above solve the problem for a known plant model dealing with uncertainties related to state perturbations and measurement noises. [8] dealt with model uncertainties using a conservative assumption of the relation between the matrix uncertainty and perturbation. In our paper, the problem of state estimation of dynamic systems with interval uncertainties will be considered by using zonotopes. An interval dynamic system [17], [18] is defined as a system where the exact values of its parameters are unknown but bounded by some intervals.

The main contribution of this paper is a new approach to determine the state estimation for dynamic systems with interval uncertainties. This is an extended and improved version of the method presented in [19] for interval dynamic systems. In order to improve the results in [19] a bisection algorithm is added to obtain the best approximation of the guaranteed state set. The proposed method is also generalized for multivariable systems with interval uncertainties. The proposed approach allows to move most of the on-line computation in the existing approaches [7], [9] by solving offline a Linear Matrix Inequality (LMI) optimization problem. The on-line computation time (singular value decomposition in [7], convex optimization in [9]) is considerably reduced and a reasonable performance of the state estimation set is obtained in comparison to existing approaches (e.g. [9]).

The paper is organized as follows. Section II reminds some basic definitions and properties of zonotopes that will be used along this paper. In Section III, the class of dynamical systems with interval uncertainties is defined. The next section presents the new approach to compute an outer bound 
of the state estimation by zonotopes for systems with interval uncertainties. In Section V, an example is proposed in order to show the advantages of the developed algorithm. Finally, some concluding remarks and perspectives are presented.

The following notations will be used throughout the paper:

- An interval: $\mathcal{I}=[a, b] \triangleq\{x: a \leq x \leq b\}$ with its center $\operatorname{mid}(\mathcal{I})=\frac{a+b}{2}$ and its radius $\operatorname{rad}(\mathcal{I})=\frac{b-a}{2}$;

- A unitary interval: $\mathbf{B}=[-1,1]$;

- The set of real compact intervals $[a, b]: \mathbb{I}$, with $a, b \in \mathbb{R}$ and $a \leq b$

- A box: $\left(\left[a_{1}, b_{1}\right], \ldots,\left[a_{n}, b_{n}\right]\right)^{T}$, which is an interval vector;

- A unitary box in $\mathbb{R}^{m}: \mathbf{B}^{m}$, which is a box composed by $m$ unitary intervals;

- An interval matrix: $[M] \in \mathbb{I}^{n \times m}$, which is a matrix whose elements are intervals. It means that each element $M_{i j}$, with $i=1, \ldots, n$ and $j=1, \ldots, m$, of this matrix is defined as $M_{i j}=\left\{m_{i j}: a_{i j} \leq m_{i j} \leq b_{i j}\right\}$. In the matrix space, this interval matrix is a hyper-rectangle and hence a convex set;

- The set of all vertices of the interval matrix [M]: $\operatorname{vert}([M])$, which denotes the set of all matrices $S=\left[s_{i j}\right]$ such that $s_{i j}=a_{i j}$ or $s_{i j}=b_{i j}$ for all $i=1, \ldots, n$ and $j=1, \ldots, m$;

- The center of an interval matrix [M]: $\operatorname{mid}([M])_{i j}=\frac{a_{i j}+b_{i j}}{2}, i=1, \ldots, n$ and $j=1, \ldots, m$;

- The radius of an interval matrix $[M]: \operatorname{rad}([M])_{i j}=$ $\frac{b_{i j}-a_{i j}}{2}, i=1, \ldots, n$ and $j=1, \ldots, m$;

- The Minkowski sum of two sets $X$ and $Y$ : $X \oplus Y \triangleq\{x+y: x \in X, y \in Y\}$;

- A strip: $\bar{S} \triangleq\left\{x \in \mathbb{R}^{n}:\left|c^{T} x-d\right| \leq \sigma\right\}$, with $c \in \mathbb{R}^{n}$ and $d, \sigma \in \mathbb{R}^{+}$;

- A positive (resp. negative) definite matrix: $M \succeq 0$ (resp. $M \preceq 0)$;

- The "row sum" diagonal matrix of a matrix $M \in \mathbb{R}^{n \times m}$ [7]: $\operatorname{rs}(M)=\operatorname{diag}\left(\tilde{m}_{i i}\right)$, with $\tilde{m}_{i i}=\sum_{j=1}^{m}\left|m_{i j}\right|$, $i=1, \ldots, n$.

\section{Fundamental Definitions AND Properties}

This section reminds some basic definitions and properties of zonotopes which are necessary to understand the concepts developed in this paper.

A zonotope is a convex symmetric polytope. A zonotope of order $m$ in $\mathbb{R}^{n}$ can be defined as the linear image of a $m$-dimensional hypercube in $\mathbb{R}^{n}$. The order $m$ is a measure of the geometrical complexity of the zonotope. Given a vector $p \in \mathbb{R}^{n}$ and a matrix $H \in \mathbb{R}^{n \times m}$, a $m$-zonotope in $\mathbb{R}^{n}$ is defined as $Z=p \oplus H \mathbf{B}^{m}=\left\{p+H z: z \in \mathbf{B}^{m}\right\}$. This is the Minkowski sum of the $m$-segments defined by $m$ columns of matrix $H$ in $\mathbb{R}^{n}$.

The $P$-radius of a zonotope $Z=p \oplus H \mathbf{B}^{m}$ is defined as $L=\max \left(\|z-p\|_{P}^{2}\right)$, with $z \in Z$. This notion is related to the ellipsoid $(x-p)^{T} P(x-p) \leq 1$.

Property 1: ([7]) Given two centered zonotopes $Z_{1}=H_{1} \mathbf{B}^{m_{1}} \in \mathbb{R}^{n}$ and $Z_{2}=H_{2} \mathbf{B}^{m_{2}} \in \mathbb{R}^{n}$, the Minkowski sum of two zonotopes is also a zonotope defined by $Z=Z_{1} \oplus Z_{2}=\left[\begin{array}{ll}H_{1} & H_{2}\end{array}\right] \mathbf{B}^{m_{1}+m_{2}}$.
Property 2: ([7]) The image of a centered zonotope $Z_{1}=H_{1} \mathbf{B}^{m_{1}} \in \mathbb{R}^{n}$ by a linear application $K$ can be computed by a standard matrix product $K \cdot Z_{1}=\left(K \cdot H_{1}\right) \mathbf{B}^{m_{1}}$.

Property 3: (Zonotope reduction) ([7], [9]) Given the zonotope $Z=p \oplus H \mathbf{B}^{m} \in \mathbb{R}^{n}$ and the interger $s$ with $n<s<m$, denote $\hat{H}$ the resulting matrix after reordering the columns of the matrix $H=\left[h_{1} \ldots h_{i} \ldots h_{m}\right]$ in decreasing order of Euclidean norm $\left(\hat{H}=\left[\hat{h}_{1} \ldots \hat{h}_{i} \ldots \hat{h}_{m}\right]\right.$ with $\left.\left\|\hat{h}_{i}\right\|_{2} \geq\left\|\hat{h}_{i+1}\right\|_{2}\right)$. Define the matrix $\hat{H}_{T}$ obtained from the first $s-n$ columns of matrix $\hat{H}$ and the matrix $\hat{H}_{Q}$ the rest of the matrix $\hat{H}$. Then the following inclusion is obtained $Z \subseteq p \oplus\left[\hat{H}_{T} \quad r s\left(\hat{H}_{Q}\right)\right] \mathbf{B}^{s}$.

This property is used in the Section IV allowing to estimate a high-order zonotope by a lower-order zonotope.

Property 4: (Zonotope inclusion) ([9]) Consider a family of zonotopes represented by $Z=p \oplus[M] \mathbf{B}^{m}$ where $p \in \mathbb{R}^{n}$ is a real vector and $[M] \in \mathbb{I}^{n \times m}$ is an interval matrix. A zonotope inclusion (denoted $\diamond(Z)$ ) is an outer approximation of this family which is defined by:

$$
\diamond(Z)=p \oplus[\operatorname{mid}([M]) \quad \operatorname{rs}(\operatorname{rad}([M]))] \mathbf{B}^{m+n}
$$

Property 5: Given an interval matrix [M] $\in \mathbb{I}^{n \times p}$ and a real matrix $N \in \mathbb{R}^{p \times q}$, the center and the radius of the interval matrix defined by the product $[M] N$ are given by $\operatorname{mid}([M] N)=\operatorname{mid}([M]) N$ and $\operatorname{rad}([M] N)=\operatorname{rad}([M])|N|$, where $|N|$ designates the absolute value of each element of $N$.

Property 6: An interval vector $[c] \in \mathbb{I}^{n}$ is defined by the zonotope $[c]=\operatorname{mid}([c]) \oplus \operatorname{rs}(\operatorname{rad}([c])) \mathbf{B}^{n}$.

\section{PROBLEM FORMULATION}

Consider the following linear discrete time variant system of the form:

$$
\left\{\begin{array}{l}
x_{k+1}=A_{k} x_{k}+\omega_{k} \\
y_{k}=c^{T} x_{k}+v_{k}
\end{array}\right.
$$

where $x_{k} \in \mathbb{R}^{n}$ is the state of the system, $y_{k} \in \mathbb{R}$ is the measured output at sample time $k$. The vector $\omega_{k} \in \mathbb{R}^{n_{\omega}}$ represents the state perturbation vector and $v_{k} \in \mathbb{R}$ is the measurement perturbation (noise, offset, etc.). The matrix $A_{k}$ is unknown but belongs to a Schur stable interval matrix [A] (all matrices in the interval matrix [A] are Schur stable) [20], [21]. This assumption is not conservative because in many applications the matrix $A_{k}$ is given by a closed-loop matrix $\tilde{A}_{k}+\tilde{B}_{k} K$, where $\tilde{A}_{k}, \tilde{B}_{k}$ are the open-loop matrices with $\tilde{A}_{k}$, resp. $\tilde{B}_{k}$ belonging to the interval matrices $[\tilde{A}]$, resp. $[\tilde{B}]$. The stabilizing controller gain $K$ can be computed by solving a LMI problem [22]. It is assumed that the uncertainties and the initial state are bounded by zonotopes: $\omega_{k} \in W, v_{k} \in V$ and $x_{0} \in X_{0}$. To simplify the computation, the center of $W$ and $V$ are assumed to be the origin. Note that if this assumption is not satisfied, a change of coordinates can be used. From the definition of a zonotope, $W$ and $V$ can be written as $W=F \mathbf{B}^{n_{\omega}}$ and $V=\sigma \mathbf{B}^{1}$, with $F \in \mathbb{R}^{n \times n_{\omega}}$ and $\sigma \in \mathbb{R}^{+}$. With these notations, the consistent state set and the exact uncertain set are defined as follows. 
Definition 1: Given the system (2) and a measured output $y_{k}$, the consistent state set at time $k$ is defined as $X_{y_{k}}=\left\{x \in \mathbb{R}^{n}:\left|c^{T} x-y_{k}\right| \leq \sigma\right\}$.

Definition 2: Consider the system (2). The exact uncertain state set $X_{k}$ is equal to the set of states that are consistent with the measured output and the initial state set $X_{0}$ : $X_{k}=\left(A_{k-1} X_{k-1} \oplus F \mathbf{B}^{n_{\omega}}\right) \cap X_{y_{k}}$, for $k \geq 1$.

Remark 1: The computation of the exact uncertain state set is a difficult task. In the literature, this set is approximated by using different geometrical forms such as polytopes, parallelotopes, ellipsoids or zonotopes. In this paper, due to its properties, a zonotopic outer approximation will be determined using the following algorithm which is similar to the Kalman filter. Suppose the state at instant $k-1$ is known to belong to the zonotope $\hat{X}_{k-1}$ and the measured output $y_{k}$ at instant $k$ is available.

\section{Algorithm 1}

1) Prediction step. Given the system (2), compute a zonotopic set $\bar{X}_{k}$ that offers a bound for the uncertain trajectory of the system by using the zonotope inclusion.

2) Measurement. Compute the consistent state set $X_{y_{k}}$ by using the measurement $y_{k}$. According to the assumption on $v_{k}$ this set can be represented by a strip as $X_{y_{k}}=\left\{x \in \mathbb{R}^{n}:\left|c^{T} x-y_{k}\right| \leq \sigma\right\}$.

3) Correction step. In order to find the state estimation set, compute an outer approximation $\hat{X}_{k}$ of the intersection between $X_{y_{k}}$ and $\bar{X}_{k}$.

To obtain a zonotope bounding the uncertain trajectory of the system, Properties 1 and 2 are used. The complexity of this zonotope is limited (from Property 3). To compute the intersection set from step 3, an optimization problem will be solved as detailed in the next section.

\section{GuARANTEED STATE INTERSECTION FOR SYSTEMS WITH INTERVAL UNCERTAINTIES}

This section states the main result of this paper. In the last step of Algorithm 1, the intersection between the zonotope $\bar{X}_{k}$ and the strip $X_{y_{k}}$ is approximated by an outer family of zonotopes (parameterized by a vector $\lambda$ ). This is resumed by the following property.

Property 7: ([9]) Given a zonotope $X=p \oplus H \mathbf{B}^{r} \subset \mathbb{R}^{n}$, a strip $S=\left\{x \in \mathbb{R}^{n}:\left|c^{T} x-d\right| \leq \sigma\right\}$ and a vector $\lambda \in \mathbb{R}^{n}$. Define a parametrized vector $\hat{p}(\lambda)=p+\lambda\left(d-c^{T} p\right) \in \mathbb{R}^{n}$ and a parametrized matrix $\hat{H}(\lambda)=\left[\left(I-\lambda c^{T}\right) H \sigma \lambda\right] \in \mathbb{R}^{n \times(m+1)}$. Then the following expression holds $X \cap S \subseteq \hat{X}(\lambda)=\hat{p}(\lambda) \oplus \hat{H}(\lambda) \mathbf{B}^{r+1}$.

In [9], there exist two methods for choosing $\lambda$, that are based either on the minimization of the segments of the zonotope or on the minimization of the volume of the intersection. The first approach results in a fast computation but with a loss of performance for the estimation, while the second approach offers more accurate results but increases the computation time because an optimization problem must be solved at each sampling time.

This section proposes a new approach (which is the main contribution of this paper) offering a good trade-off between performance and computation time. A different criterion based on the minimization of the P-radius of a zonotope is used to compute the vector $\lambda$ from Property 7 in order to combine the advantages of the two mentioned methods.

Denote an outer approximation of the state set $\hat{X}_{k}=\hat{p}_{k} \oplus \hat{H}_{k} \mathbf{B}^{r}$ at the time instant $k$ and the measured output $y_{k+1}$ at the instant $k+1$. Then the predicted state set at the next time instant $\bar{X}_{k+1}$ can be computed using (2), Property 1 and Property 2:

$\bar{X}_{k+1}=A_{k} \hat{p}_{k} \oplus\left[A_{k} \hat{H}_{k} \quad F\right] \mathbf{B}^{r+n_{\omega}}=\bar{p}_{k+1} \oplus \bar{H}_{k+1} \mathbf{B}^{r+n_{\omega}}$

An outer approximation of the intersection (the exact estimation set) between the predicted state set and the strip (which represents the measured output) can be computed based on Property 7. This leads to:

$$
\hat{X}_{k+1}(\lambda)=\hat{p}_{k+1}(\lambda) \oplus \hat{H}_{k+1}(\lambda) \mathbf{B}^{r+n_{\omega}+1}
$$

with the parametrized vector $\hat{p}_{k+1}(\lambda)=A_{k} \hat{p}_{k}+$ $\lambda\left(y_{k+1}-c^{T} A_{k} \hat{p}_{k}\right)$ and the parametrized matrix

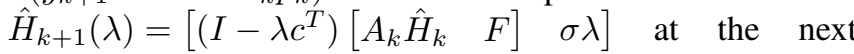
time instant $k+1$.

The idea is to compute a symmetric positive definite matrix $P=P^{T} \succeq 0$ and a vector $\lambda$ such that the $P$-radius of the zonotopic state estimation set is decreased. This means that the zonotopic state estimation set is contracted in time.

Denote the $P$-radius of the state estimation set at instant $k$ as $L_{k}=\max \left(\left\|x-\hat{p}_{k}\right\|_{P}^{2}\right)$ or $L_{k}=\max _{z} \beta\left\|\hat{H}_{k} z\right\|_{P}^{2}$. This condition can be expressed in a mathematical formulation as follows:

$$
L_{k+1} \leq \beta L_{k}+\max _{\omega}\|F \omega\|_{2}^{2}+\sigma^{2}
$$

or this is equivalent to:

$$
\max _{\hat{z}}\left\|\hat{H}_{k+1} \hat{z}\right\|_{P}^{2} \leq \max _{z} \beta\left\|\hat{H}_{k} z\right\|_{P}^{2}+\max _{\omega}\|F \omega\|_{2}^{2}+\sigma^{2}
$$

with $\hat{z}=\left[\begin{array}{c}z \\ \omega \\ \eta\end{array}\right] \in \mathbf{B}^{r+n_{\omega}+1}, z \in \mathbf{B}^{r}, \omega \in \mathbf{B}^{n_{\omega}}, \eta \in \mathbf{B}^{1}$, and $\beta \in[0,1)$.

Using the explicit form of $\hat{z}$, the following expression is a sufficient condition to satify the expression (6) (reverse triangle inequality):

$$
\max _{z, \omega, \eta}\left(\left\|\hat{H}_{k+1}\left[\begin{array}{c}
z \\
\omega \\
\eta
\end{array}\right]\right\|_{P}^{2}-\beta\left\|\hat{H}_{k} z\right\|_{P}^{2}-\|F \omega\|_{2}^{2}-\sigma^{2}\right) \leq 0
$$

Then (7) is equivalent to:

$$
\hat{z}^{T} \hat{H}_{k+1}^{T} P \hat{H}_{k+1} \hat{z}-\beta z^{T} \hat{H}_{k}^{T} P \hat{H}_{k} z-\omega^{T} F^{T} F \omega-\sigma^{2} \leq 0 \quad \forall \hat{z}
$$

Define $\eta \in \mathbf{B}^{1}$ so that $\|\eta\|_{\infty} \leq 1$. As a consequence, the following inequality is true $\sigma^{2}\left(1-\eta^{2}\right) \geq 0$. Adding this term to the left hand size of (8) leads to a sufficient condition:

$$
\begin{array}{r}
\hat{z}^{T} \hat{H}_{k+1}^{T} P \hat{H}_{k+1} \hat{z}-\beta \tilde{z}^{T} \hat{H}_{k}^{T} P \hat{H}_{k} \tilde{z}-\tilde{\omega}^{T} F^{T} F \omega-\sigma^{2}+ \\
+\sigma^{2}\left(1-\eta^{2}\right) \leq 0
\end{array}
$$


This is equivalent to:

$\hat{z}^{T} \hat{H}_{k+1}^{T} P \hat{H}_{k+1} \hat{z}-\beta z^{T} \hat{H}_{k}^{T} P \hat{H}_{k} z-\omega^{T} F^{T} F \omega-\sigma^{2} \eta^{2} \leq 0$

With the notation $\theta=\hat{H}_{k} z \in \mathbb{R}^{n \times 1}$, a matrix formulation of the inequality (10) is the following:

$$
\left[\begin{array}{c}
\theta \\
\omega \\
\eta
\end{array}\right]^{T}\left[\begin{array}{ccc}
A_{11} & A_{12} & A_{13} \\
* & A_{22} & A_{23} \\
* & * & A_{33}
\end{array}\right]\left[\begin{array}{c}
\theta \\
\omega \\
\eta
\end{array}\right] \leq 0, \quad \forall\left[\begin{array}{c}
\theta \\
\omega \\
\eta
\end{array}\right] \neq 0
$$

with the symbol' $*$ ' used for the corresponding transposed terms and the additional notations:

$$
\left\{\begin{array}{l}
A_{11}=\left(\left(I-\lambda c^{T}\right) A_{k}\right)^{T} P\left(\left(I-\lambda c^{T}\right) A_{k}\right)-\beta P \\
A_{12}=\left(\left(I-\lambda c^{T}\right) A_{k}\right)^{T} P\left(I-\lambda c^{T}\right) F \\
A_{13}=\left(\left(I-\lambda c^{T}\right) A_{k}\right)^{T} P \sigma \lambda \\
A_{22}=\left(\left(I-\lambda c^{T}\right) F\right)^{T} P\left(I-\lambda c^{T}\right) F-F^{T} F \\
A_{23}=\left(\left(I-\lambda c^{T}\right) F\right)^{T} P \sigma \lambda \\
A_{33}=\sigma^{2} \lambda^{T} P \lambda-\sigma^{2} .
\end{array}\right.
$$

Using the definition of a positive-definite matrix, the expression (11) is equivalent to the following matrix inequality:

$$
\left[\begin{array}{ccc}
A_{11} & A_{12} & A_{13} \\
* & A_{22} & A_{23} \\
* & * & A_{33}
\end{array}\right] \preceq 0, \quad \forall\left[\begin{array}{c}
\theta \\
\omega \\
\eta
\end{array}\right] \neq 0
$$

which is further equivalent to:

$$
\left[\begin{array}{ccc}
-A_{11} & -A_{12} & -A_{13} \\
* & -A_{22} & -A_{23} \\
* & * & -A_{33}
\end{array}\right] \succeq 0, \quad \forall\left[\begin{array}{c}
\theta \\
\omega \\
\eta
\end{array}\right] \neq 0
$$

Let $\operatorname{vert}([A])$ be the set of all vertices of $[A]$. As $[A]$ is a convex set, if (14) is true on each vertex of $[A]$, then it is true for all $A_{k} \in[A]$. This is a generalization of the technique in [19] where a fixed known matrix $A$ is considered instead of $A_{k}$. Using the explicit notations (12) and doing some manipulations in (14) lead to a BMI (Bilinear Matrix Inequality) problem:

$$
\begin{aligned}
& {\left[\begin{array}{ccc}
\beta P & 0 & 0 \\
* & F^{T} F & 0 \\
* & * & \sigma^{2}
\end{array}\right]-} \\
- & {\left[\begin{array}{c}
\left(S_{i}^{T}-S_{i}^{T} c \lambda^{T}\right) P \\
\left.\left(F^{T}-F^{T} c \lambda^{T}\right) P\right) \\
\\
\lambda^{T} P \sigma
\end{array}\right] P^{-1}\left[\begin{array}{c}
\left(S_{i}^{T}-S_{i}^{T} c \lambda^{T}\right) P \\
\left.\left(F^{T}-F^{T} c \lambda^{T}\right) P\right) \\
\lambda^{T} P \sigma
\end{array}\right]^{T} \succeq 0 }
\end{aligned}
$$

where $S_{i}, i=1, \ldots, 2^{q}$ are the vertices of the interval matrix $[A]$ and $q$ is the number of interval elements of $[A]$.

Applying the Schur complement [23], it is equivalent to the following BMI:

$$
\left[\begin{array}{cccc}
\beta P & 0 & 0 & S_{i}^{T} P-S_{i}^{T} c Y^{T} \\
* & F^{T} F & 0 & F^{T} P-F^{T} c Y^{T} \\
* & * & \sigma^{2} & Y^{T} \sigma \\
* & * & * & P
\end{array}\right] \succeq 0
$$

with $\beta, P$ and $\lambda$ as decision variables.
As the 2-norm is a convex function and $W$ is a convex set the constant term const $=\max _{\omega}\|F \omega\|_{2}^{2}$ can be easily computed. Then the condition (6) can be written as $L_{k+1} \leq$ $\beta L_{k}+$ const $+\sigma^{2}$, which at infinity is equivalent to:

$$
L_{\infty}=\beta L_{\infty}+\text { const }+\sigma^{2}
$$

or even

$$
L_{\infty}=\frac{\sigma^{2}+\text { const }}{1-\beta}
$$

Consider now an ellipsoid $\left\{x: x^{T} P x \leq \frac{\sigma^{2}+\text { const }}{1-\beta}\right\}$ or the normalized ellipsoid $\left\{x: x^{T} \frac{(1-\beta) P}{\sigma^{2}+\text { const }} x \leq 1\right\}$. To minimize the $P$-radius (which is $L_{\infty}$ ) of the zonotope, the ellipsoid with the smallest diameter must be found ([23]). This is equivalent to solve an EVP (eigenvalue problem). The following algorithm synthesizes this problem.

\section{Algorithm 2}

Using the bisection algorithm [24], find the smallest value of $\beta \in[0,1)$ such that the following optimization problem is feasible:

$$
\max _{\tau, P, Y} \tau
$$

subject to the LMIs:

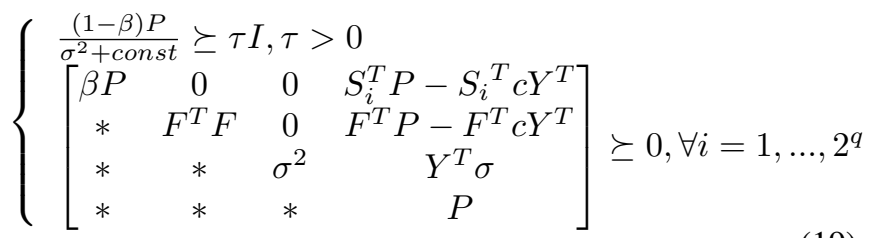

with the change of variable $Y=P \lambda$. The decision variables are: $P=P^{T} \in \mathbb{R}^{n \times n}, Y \in \mathbb{R}^{n}$ and $\tau \in \mathbb{R}$. The total number of scalar decision variables is $\frac{n(n+1)}{2}+n+1=\frac{n^{2}+3 n+2}{2}$. Note that this LMI optimization problem is solved off-line allowing to reduce the overall computation time compared to the algorithms developed in [9]. Moreover, in order to decrease the domain complexity, a reduction step based on Property 3 is implemented to approximate the obtained highorder zonotope by a lower-order zonotope. In addition, to reduce the computational complexity when dealing with high order interval matrices, the methods developed in [22] can be used.

To summarize, the guaranteed state estimation using the P-radius minimization can be implemented in two phases. Firstly, an optimal value of vector $\lambda$ is computed using Algorithm 2. Secondly, for $k=1, \ldots, N$ iterations, Algorithm 1 is implemented to compute the zonotopic state estimation set introducing the value of $\lambda$ into the correction step of Algorithm 1. Note that after computing the vector $\lambda$, the decrement speed depends only on the matrix $A_{k}$. The bisection algorithm is used to obtain the smallest value of $\beta$ which allows that the guaranteed state estimation set decreases quickly and has a better performance in comparison to [19].

As $A_{k}$ is unknown but belong to the interval matrix [A], in each iteration $\bar{X}_{k}$ is computed by a zonotopic outer approximation using Properties 1 to 6 , as detailed in Appendix:

$$
\bar{X}_{k}=\operatorname{mid}([A]) \hat{p}_{k} \oplus G \mathbf{B}^{l}
$$


with $l=r+2 n+n_{\omega}$ and the notation $G=\left[\begin{array}{llll}\operatorname{mid}([A]) \hat{H}_{k} & F & r s\left(r_{A H_{k}}\right) & r s\left(r_{A p_{k}}\right)\end{array}\right] \quad$ where $r_{A H_{k}}=\operatorname{rad}([A])\left|\hat{H}_{k}\right|$ and $\left.r_{A p_{k}}=\operatorname{rad}([A])\left|\hat{p}_{k}\right|\right)$.

Since the matrices $A_{k}$ are stable, the states of the system converge to the origin and thus the term $\operatorname{rad}([A])\left|\hat{p}_{k}\right|$ is bounded and time decreasing. Then the contractive property of the estimation set is always preserved.

Remark 2: For multivariable systems the procedure can be repeated for each element of the measurement vector $y_{k}$.

\section{ILLUSTRATIVE EXAMPLE}

In this section, the same linear discrete time-variant system as described in [9] is used for further comparisons:

$$
\left\{\begin{array}{l}
x_{k+1}=\left[\begin{array}{cc}
0 & -0.5 \\
1 & 1+0.3 \delta_{k}
\end{array}\right] x_{k}+0.02\left[\begin{array}{c}
-6 \\
1
\end{array}\right] \omega_{k} \\
y_{k}=\left[\begin{array}{ll}
-2 & 1
\end{array}\right] x_{k}+0.2 v_{k}
\end{array}\right.
$$

with $\delta_{k} \leq 1,\left\|v_{k}\right\|_{\infty} \leq 1,\left\|\omega_{k}\right\|_{\infty} \leq 1$ generated by random functions. The initial state $x_{0}$ belongs to the box $3 \mathbf{B}^{2}$ and the order of the zonotopes is limited to $m \leq 20$ in the interest of a fast simulation. A simple second order system is chosen in order to facilitate the graphical visualization.

The evolution of the state estimation set is shown in Fig 1. This set is an outer approximation of the intersection between the predicted state set $\bar{X}_{k}$ and the measurement $X_{y_{k}}$. The size of this set is decreased at each iteration.

To show the advantage of the $P$-radius minimization approach, in this paper a performance comparison (the bound's width of $x_{1_{k}}$ ) to the existing approaches is done (Fig 2\&3). The continuous lines (resp. the dotted lines and the dash dotted lines) are the bounds of $x_{1_{k}}$ obtained by the $P$ radius minimization approach (resp. the segment minimization approach and the volume minimization approach). The exact states $x_{1_{k}}$ at each iteration are represented by the stars. These stars lie between the bounds of $x_{1_{k}}$ obtained by the set membership estimation approach which confirms a good estimation. For a more clear representation, the bound's width are compared also in percent values (Fig 3). The bound's width obtained by the segment minimization is considered as 100. Then this is compared to the bound's width obtained by the $P$-radius approach and the volume minimization approach. The simulation results confirm a better performance $(10 \%)$ of the $P$-radius than the segment minimization approach and a comparable performance of the proposed method compared to the volume minization approach.

The comparison of computation time between different algorithms is shown in Table I. The LMI optimization from Algorithm 2 is solved using the LMI toolbox of Matlab ${ }^{\circledR}$. If the off-line computation time is not taken into account, the $P$-radius approach offers the same performance as the segment minimization and 4 times better than the volume minimization approach. All the simulations are performed on an Intel Core 2 Duo E8500 3.16 GHz configuration.

To conclude, the segment minimization approach offers a short computation time with a loss of performance. The

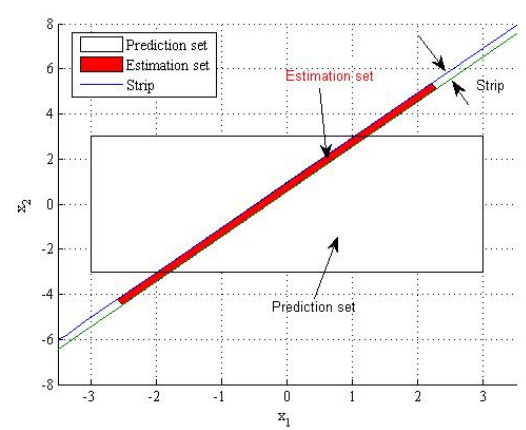

(a) $k=1$

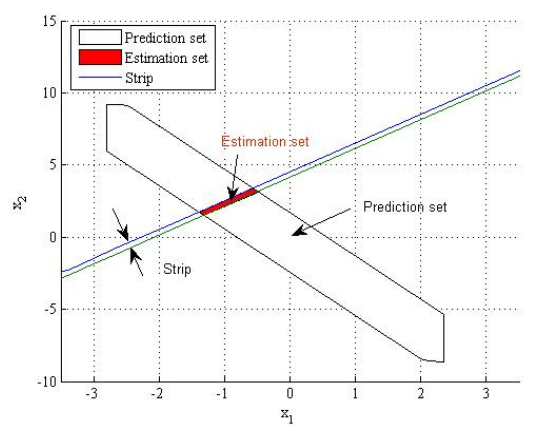

(b) $k=2$

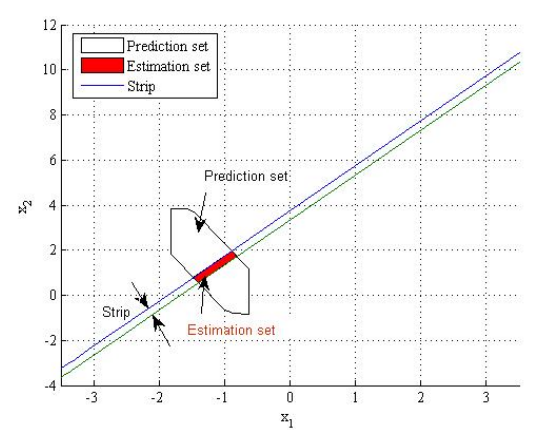

(c) $k=3$

Fig. 1. Intersection $\hat{X}_{k}$ between the predicted state set $\bar{X}_{k}$ and the measurement $X_{y_{k}}$

volume minimization approach allows to obtain a better performance but the computation is more complex. The $P$ radius approach allows to combine the advantage of the two existing approaches: fast computation time and good performance.

\section{CONCLUSION}

In this paper a new zonotopic outer-bounding for the state estimation of a linear discrete time-variant system with model interval uncertainties has been developed. The proposed method computes a set of all the states that are consistent with the measured output, the bounded noise, the bounded perturbation and the model interval uncertainty. Based on the minimization of the P-radius of a zonotope, the size of this zonotope is decreased. The new method offers a good trade-off between the performance and the 


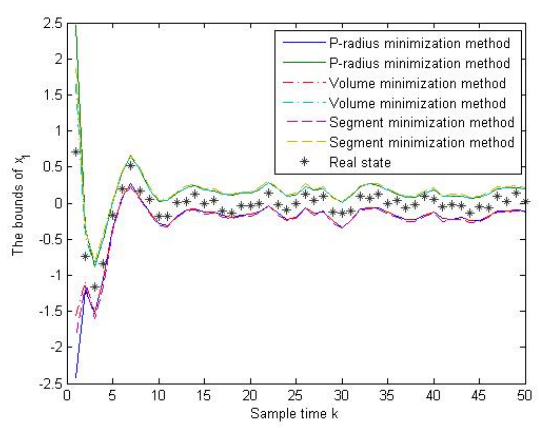

Fig. 2. The bounds of $x_{1}$ obtained by different methods

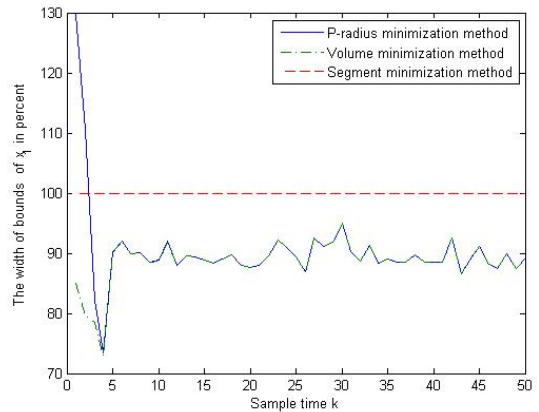

Fig. 3. Comparison of the bound's width of $x_{1}$ obtained by different methods

computation time compared to existing methods. An outputfeedback control of the linear discrete time-variant systems via set-membership state estimation will be considered in future work.

\section{APPENDIX}

This Appendix proposes the proof of the expression (20). The starting point is given by equation (3): $\bar{X}_{k+1}=A_{k} \hat{p}_{k} \oplus\left[\begin{array}{ll}A_{k} \hat{H}_{k} & F\end{array}\right] \mathbf{B}^{r+n_{\omega}}$. As $A_{k}$ is bounded by the interval matrix $[A]$, an outer approximation of $\bar{X}_{k+1}$ can be obtained by $[A] \hat{p}_{k} \oplus\left[[A] \hat{H}_{k} \quad F\right] \mathbf{B}^{r+n_{\omega}}$.

Using Property 6, the following expression is true: $[A] p \in \operatorname{mid}([A]) \hat{p}_{k} \oplus \operatorname{rs}\left(\operatorname{rad}([A])\left|\hat{p}_{k}\right|\right) \mathbf{B}^{n}$.

In addition, Properties 4 and 5 imply that $[A] \hat{H}_{k} \mathbf{B}^{r} \in\left[\operatorname{mid}([A]) \hat{H}_{k} \quad \operatorname{rs}\left(\operatorname{rad}([A])\left|\hat{H}_{k}\right|\right)\right] \mathbf{B}^{r+n}$.

The Minkowski sum of the last two expressions leads to: $\operatorname{mid}([A]) \hat{p}_{k} \oplus \operatorname{rs}\left(\operatorname{rad}([A])\left|\hat{p}_{k}\right|\right) \mathbf{B}^{n} \oplus$

$\left.\oplus\left[\operatorname{mid}([A]) \hat{H}_{k} \quad \operatorname{rs}\left(\operatorname{rad}([A])\left|\hat{H}_{k}\right|\right)\right)\right] \mathbf{B}^{r+n}$.

Therefore the zonotopic outer approximation of $\bar{X}_{k+1}$ is $\operatorname{mid}([A]) \hat{p}_{k} \oplus G \mathbf{B}^{l}$, with $l=r+2 n+n_{\omega}$ and the matrix $G$

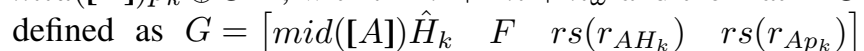
where $r_{A H_{k}}=\operatorname{rad}([A])\left|\hat{H}_{k}\right|$ and $\left.r_{A p_{k}}=\operatorname{rad}([A])\left|\hat{p}_{k}\right|\right)$.

\section{REFERENCES}

[1] R. E. Kalman, "A new approach to linear filtering and prediction problems," Transactions of the ASME-Journal of Basic Engineering, vol. 82, no. Series D, pp. 35-45, 1960.

[2] F. C. Schweppe, "Recursive state estimation: Unknown but bounded errors and system inputs," IEEE Trans. Automat. Contr., vol. 13(1), pp. 22-28, 1968.
TABLE I

COMPUTATION TIME AFTER 50 SAMPLE TIMES

\begin{tabular}{lc} 
Algorithm & Time(second) \\
\hline Segment minimization & 0.0312 \\
Presented algorithm (without LMI optimization) & 0.0312 \\
Presented algorithm (with LMI optimization included) & 2.1060 \\
Volume minimization & 8.7517 \\
\hline
\end{tabular}

[3] S. H. Witsenhausen, "Sets of possible states of linear systems given perturbed observations," IEEE Trans. Automat. Contr., vol. 13, pp. 556-558, 1968.

[4] D. P. Bertsekas and I. B. Rhodes, "Recursive state estimation for a setmembership description of uncertainty," IEEE Trans. Automat. Contr. vol. 16(2), pp. 117-128, 1971.

[5] E. Walter and H. Piet-Lahanier, "Exact recursive polyhedral description of the feasible parameter set for bounded-error models," IEEE Trans. Automat. Contr., vol. 34(8), pp. 911-915, 1989.

[6] A. Vicino and G. Zappa, "Sequential approximation of feasible parameter sets for identification with set membership uncertainty," IEEE Trans. Automat. Contr., vol. 41, pp. 774-785, 1996.

[7] C. Combastel, "A state bounding observer based on zonotopes," in Proc. of European Control Conference, Cambridge, UK, 2003.

[8] B. T. Polyak, S. A. Nazin, C. Durieu, and E. Walter, "Ellipsoidal parameter or state estimation under model uncertainty," Automatica, vol. 40, pp. 1171-1179, 2004.

[9] T. Alamo, J. M. Bravo, and E. F. Camacho, "Guaranteed state estimation by zonotopes," Automatica, vol. 41, pp. 1035-1043, 2005.

[10] M. Althoff, O. Stursberg, and M. Buss, "Reachability analysis of linear systems with uncertain parameters and inputs," in Proc. of the 46th IEEE Conference on Decision and Control, vol. 41, 2007, pp. 726732, New Orleans, LA, USA.

[11] A. B. Kurzhanski and I. Vályi, Ellipsoidal calculus for estimation and control. Birkhaüser Boston, 1996.

[12] W. Kühn, "Rigorously computed orbits of dynamical systems without the wrapping effect," Computing, vol. 61, pp. 47-67, 1998.

[13] A. B. Kurzhanski and P. Varaiya, "On ellipsoidal techniques for reachability analysis. Part I: External approximations," Optimization Methods and Software, vol. 17, pp. 177-206, 2001.

[14] L. J. Guibas, A. Nguyen, and L. Zhang, "Zonotopes as bounding volume," in Proc. of the Symposium on Discrete Algorithm, 2005, pp. $803-812$.

[15] A. Ingimundarson, J. M. Bravo, V. Puig, T. Alamo, and P. Guerra, "Robust fault detection using zonotope-based set-membership consistency test," International Journal of Adaptive Control and Signal Processing, vol. 23(4), pp. 311-330, 2008.

[16] A. Lalami, "Diagonostic et approaches ensemblistes base des zonotopes," Ph.D. dissertation, Université de Cergy-Pontoise (in French), 2008.

[17] L. Jaulin, M. Kieffer, O. Didrit, and E. Walter, Applied Interval Analysis. Springer, 2001.

[18] S. Khadroui, M. Rakotondrabe, and P. Lutz, "Robust control for a class of interval model: application to the force control of piezoelectric cantilevers," in Proc. of the 49th IEEE Conference on Decision and Control, 2010, Atlanta, USA.

[19] V. Le, T. Alamo, E. Camacho, C. Stoica, and D. Dumur, "A new approach for guaranteed state estimation by zonotopes," in Proc. of the 18th World Congress IFAC, Milan, Italy, 2011.

[20] M. Mansour, "Simplified sufficient conditions for the asymptotic stability of interval matrices," International Journal of Control, vol. 50, no. 1, pp. 443-444, 1989.

[21] O. Pastravanu and M. Voicu, "Necessary and sufficient conditions for componentwise stability of interval matrix systems," IEEE Trans. Automat. Contr., vol. 49(6), pp. 1016-1021, 2004.

[22] T. Alamo, R. Tempo, D. R. Ramírez, and E. F. Camacho, "A new vertex result for robustness problems with interval matrix uncertainty," Systems and Control Letters, vol. 57, pp. 474-481, 2008.

[23] S. Boyd, L. E. Ghaoui, E. Feron, and V. Balakrishnan, Linear Matrix Inequalities in System and Control Theory. Philadelphia: SIAM, 1994.

[24] R. L. Burden and J. D. Faires, Numerical Analysis. Brooks Cole, 2000 